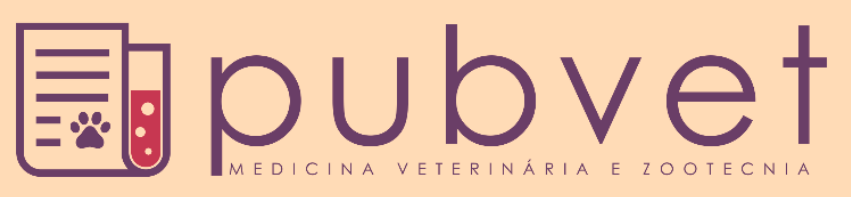

https://doi.org/10.31533/pubvet.v14n6a584.1-4

\title{
Persistência do ducto arterioso em um bovino
}

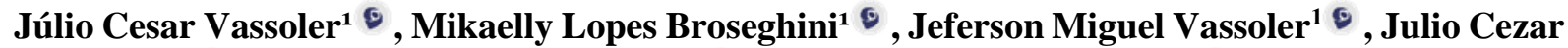 \\ Menegassi $^{10}$, Solimar Felipe $\operatorname{Contarini}^{1}{ }^{\circ}$, Luiz Alexandre $\operatorname{Moscon}^{2}{ }^{\circ}$, Mariana Caetano \\ Teixeira $^{30}$, Diogo Almeida Rondon ${ }^{2}$, Clairton Marcolongo Pereira ${ }^{4 *} \bullet$ \\ ${ }^{I}$ Graduandos de Medicina Veterinária, Centro Universitário do Espírito Santo - UNESC, Colatina - ES Brasil \\ ${ }^{2}$ Professor assistente, Hospital Veterinário "Joaquim Rossi", Faculdade de Medicina Veterinária, UNESC, Colatina -ES Brasil \\ ${ }^{3}$ Professora assistente, Centro Universitário Ritter dos Reis - UniRitter, Porto Alegre-RS Brasil. \\ ${ }^{4}$ Professor assistente, Laboratório de Patologia Veterinária, Faculdade de Medicina Veterinária, UNESC, Colatina -ES Brasil \\ *Autor para correspondência, E-mail: clairton.marcolongo@terra.com.br
}

Resumo. O ducto arterioso é uma estrutura que liga o arco pulmonar à aorta durante a vida fetal dos mamíferos, permitindo que o sangue arterial contorne o pulmão ainda não expandido. A ausência do fechamento desse canal caracteriza a persistência do ducto arterioso. O objetivo deste trabalho foi descrever um caso de persistência do ducto arterioso (PDA) em uma bezerra de raça mestiça, de quatro dias de idade. O animal apresentava apatia, decúbito e anorexia. O quadro clínico evoluiu para dispneia severa e morte do animal. Foi realizada necropsia na qual observou-se PDA. Tem sido mencionado que a PDA é infrequente em ruminantes, quando comparada com cães. Entretanto, alguns autores sugerem que a escassez de diagnóstico em grandes animais, principalmente em ruminantes, faz com que essa doença se enquadre como rara. Em seres humanos e em pequenos animais o tratamento de PDA é cirúrgico. Entretanto, não há relatos desse tratamento para bovinos. PDA em bezerros deve ser incluída no diagnóstico diferencial de animais com severa dispneia logo após o nascimento.

Palavras chave: bezerro, dispneia neonatal, necropsia, persistência do ducto arterioso

\section{Persistence of the ductus arteriosus in heifer}

\begin{abstract}
The ductus arteriosus is a structure that connects the pulmonary arch to the aorta during the fetal life of mammals. This ductus allows arterial blood to bypass the unexpanded lung. The absence of closure of this channel characterizes the persistence of the ductus arteriosus. The aim of this study was to describe a case of persistent ductus arteriosus (PDA) in a four-day-old crossbred heifer. The animal had apathy, decubitus and anorexia. The clinical signs evolved to severe dyspnea and death of the animal. Necropsy was performed in which PDA was observed. It has been mentioned that PDA is uncommon in ruminants when compared to dogs. However, some authors suggest that the lack of diagnosis in large animals, especially in ruminants, makes this disease a rare condition. In humans and small animals, the treatment of PDA is surgical. However, there are no reports of this treatment for cattle. PDA in calves should be included in the differential diagnosis of animals with severe dyspnea shortly after birth.
\end{abstract}

Keywords: heifer, neonatal dyspnea, necropsy, persistent ductus arteriosus

\section{Persistencia del conducto arterioso en un bovino}

Resumen. El conducto arterioso es una estructura que conecta el arco pulmonar con la aorta durante la vida fetal de los mamíferos, lo que permite que la sangre arterial evite el pulmón no expandido. La ausencia de cierre de este canal caracteriza la persistencia del 
conducto arterioso. El objetivo de este estudio fue describir un caso de conducto arterioso persistente (PDA) en una becerra cruzada de cuatro días de edad. El animal tenía apatía, decúbito y anorexia. El cuadro clínico evolucionó a disnea severa y muerte del animal. Se realizó una necropsia en la que se observó PDA. Se ha mencionado que el PDA es poco común en rumiantes en comparación con los perros. Sin embargo, algunos autores sugieren que la falta de diagnóstico en animales grandes, especialmente en rumiantes, hace que esta enfermedad sea una condición rara. En humanos y animales pequeños, el tratamiento del PDA es quirúrgico, a partir de la ligadura del conducto. Sin embargo, no hay informes de este tratamiento para el ganado bovino. La PDA en terneros debe incluirse en el diagnóstico diferencial de animales con disnea severa poco después del nacimiento.

Palabras clave: becerro, disnea neonatal, necropsia, conducto arterioso persistente

\section{Introdução}

O ducto arterioso é uma estrutura que liga o arco pulmonar à aorta durante a vida fetal dos mamíferos, permitindo que o sangue arterial contorne o pulmão ainda não expandido, já que a hematose ocorre na placenta e os pulmões necessitam de um pequeno volume sanguíneo (Ecco et al., 2008; Gournay, 2011). Após o nascimento, os pulmões são preenchidos por ar o que leva a queda da resistência ao fluxo sanguíneo no órgão, enquanto a pressão na aorta é elevada em consequência da rápida interrupção do fluxo anteriormente originado da placenta (Santos \& Alessi, 2016). Dessa forma, ocorrerá interrupção do fluxo através do ducto e, posteriormente, seu fechamento anatômico (Ecco et al., 2008). Em algumas espécies, é comum que o ducto permaneça aberto algumas horas após o nascimento, mas em ruminantes é considerado anormal (Dehkordi \& Hoseini, 2016).

A ausência do fechamento desse canal caracteriza a persistência do ducto arterioso (PDA), que permite que o sangue presente na artéria aorta alcance a artéria pulmonar (Santos \& Alessi, 2016). Essa patologia raramente é observada isoladamente em grandes animais, presente na maioria das vezes com outras patologias cardíacas (Dehkordi \& Hoseini, 2016).

Os sinais clínicos mais comumente observados são dispneia, ganho de peso reduzido e morte súbita de animais jovens (West, 1988). O objetivo deste estudo é relatar um caso de persistência do ducto arterioso em uma bezerra de raça mestiça no noroeste do Estado do Espírito Santo, Brasil.

\section{Relato de caso}

Foi atendido no Hospital Veterinário do Centro Universitário do Espírito Santo (UNESC), um animal da espécie bovina, fêmea, sem raça definida, pelagem preta, com $30 \mathrm{~kg}$, filha de touro da raça tabapuã com vaca mestiça. A mãe tinha boa condição corporal e era vacinada contra brucelose, raiva, carbúnculo hemático e sintomático e febre aftosa. O animal estava com o controle de ectoparasitas em dia e esta era sua $8^{\mathrm{a}}$ cria. A bezerra pertencia a uma propriedade localizada no noroeste do Espírito Santo, Brasil, que cria bovinos de corte.

Após o nascimento, a bezerra apresentou apatia, decúbito e parou de se alimentar (Figura 1). O quadro clínico evoluiu para dispneia, com frequência cardíaca de 100 batimentos por minuto e frequência respiratória de 60 movimentos por minuto. A temperatura retal era de $39,4^{\circ} \mathrm{C}$. Além disso, as mucosas oculares, oral e vaginal apresentavam-se pálidas. O bovino ainda manifestava ingurgitamentos das jugulares, comprovado pela prova do garrote.

O animal morreu quatro dias após o nascimento e foi necropsiado. Na necropsia, foi observado coração globoso, ducto arterioso persistente (Figuras 2 A e B) e hipertrofia cardíaca direita. Nos pulmões havia espuma nos brônquios e bronquíolos principais. Nos demais órgãos não foram observadas alterações.

\section{Discussão}

O diagnóstico de persistência do ducto arterioso baseou-se na observação do ducto persistente durante a necropsia e nos sinais clínicos do animal. Tem sido mencionado que a PDA é infrequente em ruminantes, quando comparada com cães. Entretanto, alguns autores sugerem que a escassez de diagnóstico em grandes animais, principalmente em ruminantes, faz com que essa doença se enquadre 
como rara (Lee \& Kim, 2004). Além disso, a dispneia presente na maioria dos casos pode ser confundida com pneumonia, o que dificulta o diagnóstico. Os sinais clínicos observados são similares a outro relato na literatura (Lee \& Kim, 2004). Tem sido observado que os bezerros afetados por PDA acabam morrendo após o início dos sinais clínicos, como foi o observado nesse caso.

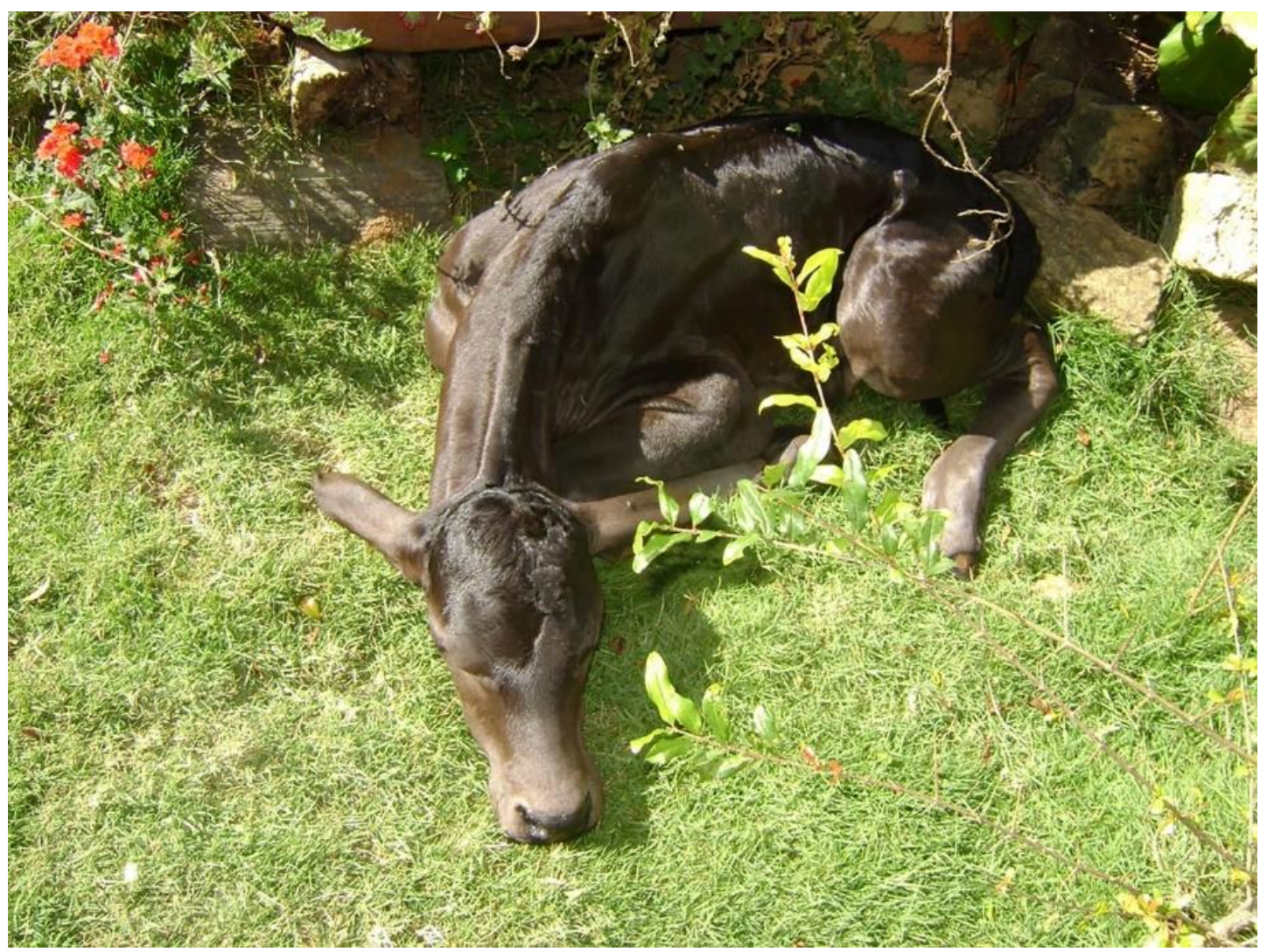

Figura 1. Persistência do ducto arterioso em um bovino. Animal apresentando apatia e decúbito.

Taquipneia, taquicardia e mucosas pálidas, apresentadas pela bezerra, ocorrem devido o desvio do fluxo sanguíneo da esquerda para direita, provocado pela PDA, reduzindo, assim, o aporte de sangue para o restante do corpo. Além disso, esse desvio sobrecarrega a artéria pulmonar e, consequentemente, acarreta uma insuficiência cardíaca congestiva direita, a qual foi comprovada pelas alterações do coração globoso, ingurgitamento das jugulares e edema pulmonar constatado no animal.

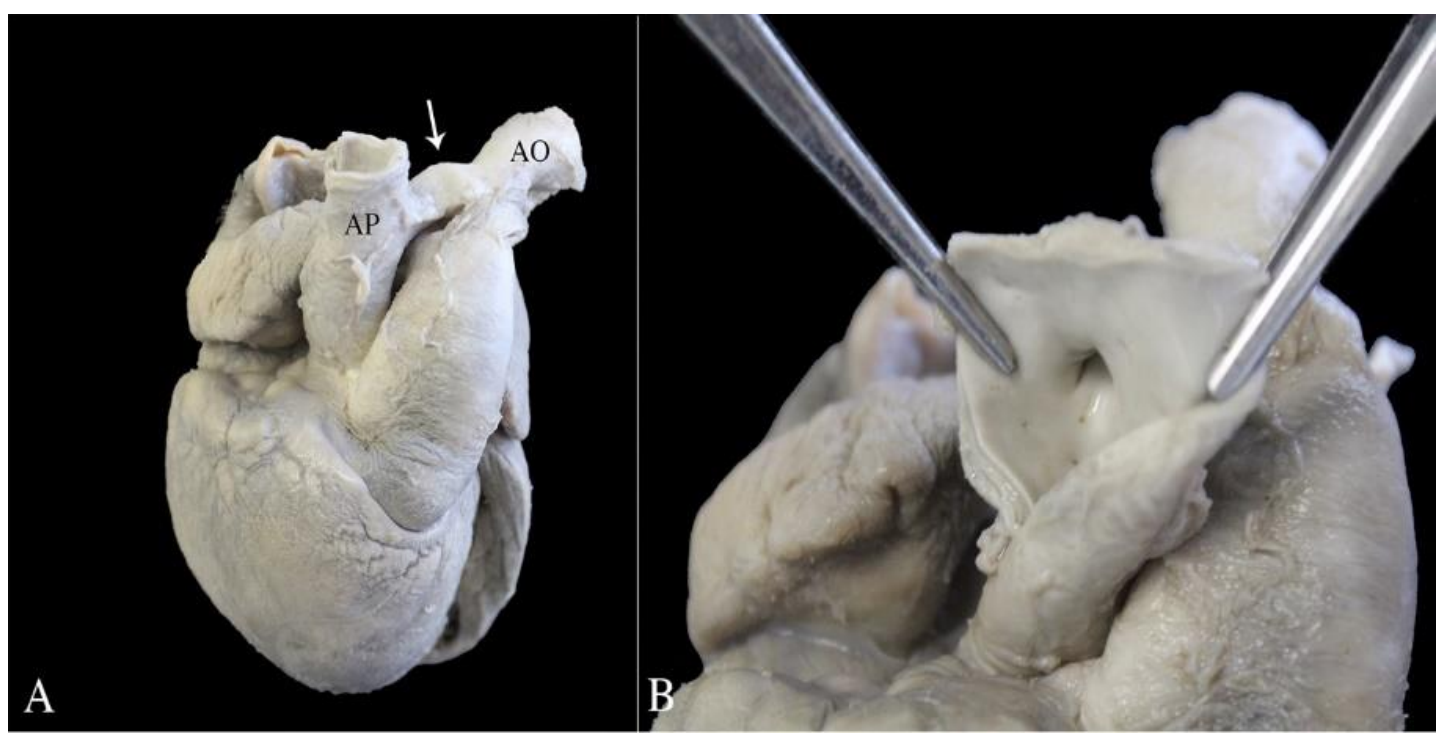

Figura 2. Persistência do ducto arterioso em um bovino. (A): Artéria pulmonar (AP) e Aorta (AO) com ducto arterioso persistente. (B): Abertura do ducto pela artéria pulmonar. 
Em bovinos ainda não está bem estabelecido a etiopatogênese da PDA. Contudo, alguns autores sugerem características hereditárias, falhas nutricionais, processos infecciosos e uso de medicamentos na gestação (Gomes et al., 2009). No presente estudo, não foi possível associar uma causa para a PDA. Provavelmente este caso trata-se de uma malformação congênita esporádica observada nesse rebanho.

Em seres humanos e em pequenos animais o tratamento de PDA é cirúrgico, feito a partir da ligadura do ducto. Entretanto, devido à dificuldade do diagnóstico e custo para os produtores, não foram encontrados relatos dessa técnica em ruminantes. O uso de medicamentos inibidores das cicloxigenasses pode ser eficiente no fechamento do ducto, já que a prostaglandina E2 é responsável por manter o canal aberto durante a vida fetal.

A persistência do ducto arterioso em bezerros deve ser incluída no diagnóstico diferencial de animais com severa dispneia logo após o nascimento. $\mathrm{O}$ ecocardiograma e a radiografia são métodos eficientes para diagnóstico em diversas espécies, entretanto, a disponibilidade de tais exames é escassa a campo (Suzuki et al., 2012).

\section{Agradecimento}

Os autores agradecem a Fundação de Amparo à Pesquisa e Inovação do Espírito Santo (FAPES) pelo apoio a este trabalho e pela bolsa concedida.

\section{Referências bibliográficas}

Dehkordi, A. J., \& Hoseini, F. (2016). Patent ductus arteriosus in a lamb: A case report. Veterinary Research Forum, 7(1), 850-858.

Ecco, R., Snel-Oliveira, M. V, Barros, R. M., \& Santos, H. L. (2008). Patent ductus arteriosus in murrah buffalos. Veterinary Pathology, 45(4), 542-545. DOI: https://doi.org/10.1354\%2Fvp.45-4-542

Gomes, V., Madureira, K. M., Contieri, M. B., Soriano, S., Pereira, K. C., \& Lunardi, L. H. (2009). Defeito do septo ventricular em bezerro neonato. Relato de caso. Ensaios e Ciência: Ciências Biológicas, Agrárias e Da Saúde, 13(2), 47-55.

Gournay, V. (2011). The ductus arteriosus: physiology, regulation, and functional and congenital anomalies. Archives of Cardiovascular Diseases, 104(11), 578-585. DOI: https://doi.org/10.1016/j.acvd.2010.06.006

Lee, B. H., \& Kim, W. G. (2004). A case of patent ductus arteriosus in a Holstein calf. Journal of Veterinary Science, 5(1), 83-84.

Santos, R. L., \& Alessi, A. C. (2016). Patologia veterinária. Roca.

Suzuki, K., Uchida, E., Schober, K. E., Niehaus, A., Rings, M. D., \& Lakritz, J. (2012). Cardiac troponin I in calves with congenital heart disease. Journal of Veterinary Internal Medicine, 26(4), 1056-1060. DOI: https://doi.org/10.1111/j.1939-1676.2012.00953.x

West, H. J. (1988). Congenital anomalies of the bovine heart. British Veterinary Journal, 144(2), 123130. DOI: https://doi.org/10.1016/0007-1935(88)90044-9

Recebido: 3 de março, 2020.

Aprovado: 12 de abril, 2020.

Disponível online: 8 julho, 2020.

Licenciamento: Este artigo é publicado na modalidade Acesso Aberto sob a licença Creative Commons Atribuição 4.0 (CC-BY 4.0), a qual permite uso irrestrito, distribuição, reprodução em qualquer meio, desde que o autor e a fonte sejam devidamente creditados. 